\section{Neu-Orientierung der Medizin}

Die Erfolge der heutigen Medizin sind offensichtlich: Die Lebenserwartung der Menschen steigt weiterhin an, die diagnostischen Methoden werden immer mehr verfeinert und täglich kommen neue, noch wirkungsvollere Behandlungsverfahren auf den Markt. Das wissenschaftliche Potenzial zur Verbesserung der Medizin ist noch lange nicht ausgeschöpft Stichwort "Human Genome Project" - und verspricht weitere, interessante Durchbrüche.

Die Medizin ist leistungsfähig wie nie zuvor. Und doch wird ihr Beitrag für Patienten und Gesellschaft durch Faktoren wie steigende Kosten, Werteunsicherheit und Probleme in der Kommunikation zum Teil erheblich eingeschränkt oder in Frage gestellt. Es ist die Rede von "Apparatemedizin", welche die Persönlichkeit der Patienten vernachlässige. In der Praxis sind Ärztinnen und Ärzte damit konfrontiert, dass viele ihrer Patientinnen und Patienten ihr Heil bzw. ihre Heilung bei nicht (schul-)medizinischen Verfahren suchen.

Ist die Medizin in der Krise? Auf Anregung von Prof. Johannes Bircher aus Bern beschloss die Schweizerische Akademie der Medizinischen Wissenschaften (SAMW) im Sommer 1999 die Lancierung des Projektes "Neu-Orientierung der Medizin". Zwei Klausurtagungen, im August 2000 und am 19./20. Januar 2001, dienen dazu, die Situation der Medizin in der Schweiz zu analysieren, das heisst,

- jene Bereiche zu identifizieren, in denen die Orientierung der gegenwärtigen Medizin als problematisch erlebt wird;

- die Mechanismen zu verstehen, welche zu Schwierigkeiten geführt haben;

- Wege zu skizzieren, auf denen eine Neu-Orientierung erreicht werden könnte.

Mittelfristig soll ein Reflexionsprozess über die sinnvollen Ziele der Medizin und deren Realisierung angestossen werden, ähnlich wie dies auf internationaler Ebene das Projekt "The Goals of Medicine" des Hastings-Centers geleistet hat. Ein mögliches Endziel besteht in der Schaffung einer politisch, wirtschaftlich und weltanschaulich unabhängigen "Denkfabrik", welche die Entwicklungen in der Medizin kontinuierlich begleitet und reflektiert, die Fragen von Verantwortungsträgern entgegennimmt und fundierte Antworten bereitstellen kann.

Das Projekt, das im kleinen Kreis begann, zeichnet sich durch sein prozesshaftes Vorgehen aus. Eine grösstmögliche Transparenz soll sicherstellen, dass dieser Prozess immer weitere Kreise mit einbezieht.
Medienmitteilungen, die direkte Anschrift und Information von Akteurinnen und Akteuren des Gesundheitswesens und der breite Versand des Zwischenberichtes zur 1. Klausurtagung (auch abrufbar auf der Website der SAMW [www.samw.ch]) dienen dazu, das Projekt einer interessierten Öffentlichkeit bekanntzumachen. Aus dem gleichen Grund erscheinen in dieser Ausgabe der Schweizerischen Ärztezeitung zwei Beiträge, welche wesentliche Aspekte der bisherigen Diskussionen wiedergeben.

Johannes Fischer, Ethikprofessor aus Zürich, plädiert für eine klare Abgrenzung von Medizin und Gesundheitswesen, dies schon allein deswegen, damit man der Medizin nicht die Probleme des Gesundheitswesens aufbürde. Da die Medizin keine Bestimmungsmacht über die normativen Kriterien besitze, glaubt er, dass die "Orientierungskrise der Medizin" in Zukunft als Dauerzustand zu betrachten sei. Im Spannungsverhältnis bzw. Ungleichgewicht zwischen der Ausrichtung der Medizin auf wissenschaftlich-technische Effizienz und ihrer Orientierung am Patienten sieht er eine wichtige Ursache für viele der diagnostizierten Probleme.

Jean Martin, Waadtländer Kantonsarzt, analysiert als Public-Health-Experte die Probleme der Medizin aus einem anderen Gesichtswinkel. Er stellt die Rechte und Pflichten des Bürgers bzw. des Patienten denjenigen der Gesellschaft gegenüber und diagnostiziert eine wachsende Kluft zwischen Ansprüchen und Ressourcen. Krankheitsbehandlung und -verhütung seien zwar die Hauptaufgaben des Gesundheitswesens, doch werde dieses auch durch andere Faktoren (politische, wirtschaftliche, kulturelle) in starkem Masse beeinflusst.

Während an der 1. Klausurtagung vom August 2000 die Probleme der Medizin und ihre Ursachen im Zentrum standen, sollen an der 2. Klausurtagung vom 19./20. Januar 2001 "Nachfrage" und "Angebot" einander gegenübergestellt sowie Wege zu einer allfälligen Neuorientierung der Medizin skizziert werden. Der nächste wichtige Termin steht bereits fest: Am Donnerstag, dem 30. August 2001, findet im Kursaal in Bern ein Symposium statt, an dem die bisherigen Ergebnisse präsentiert sowie mögliche Lösungsvorschläge zur Diskussion gestellt werden sollen. Die Teilnehmerinnen und Teilnehmer - Ärztinnen und Ärzte, Vertreterinnen und Vertreter der Pflege und weiterer medizinischer Berufe - sind dabei eingeladen, das Projekt der SAMW auf eine neue, breitere Basis zu stellen und die Neu-Orientierung der Medizin zu ihrem eigenen Anliegen zu machen.

Dr. med. Hermann Amstad Stv. Generalsekretär SAMW 